\title{
O USO DE TECNOLOGIAS DIGITAIS EM UMA INSTITUIÇÃO DE ENSINO SUPERIOR: APONTAMENTOS SOBRE A SAÚDE DO JOVEM
}

\section{Ana Luisa Martins Rosa}

Psicóloga; Bolsista do Programa de Bolsas de Iniciação Científica do Cesumar (PROBIC); Pós graduanda em Saúde Mental, Psicopatologias e Atenção Psicossocial pelo Centro Universitário de Maringá (UNICESU. MAR), Maringá (PR), Brasil.

\section{Eduardo Chierrito de Arruda}

Psicólogo; Mestrando no Programa de Pós-graduação em Tecnologias Limpas do Centro Universitário de Maringá (UNICESUMAR), Maringá (PR), Brasil.

\section{Ana Beatriz Tozzo Martins}

Doutor em Métodos Numéricos em Engenharia pela Universidade Federal do Paraná (UFPR); Docente titular da Universidade Estadual de Maringá (UEM), Maringá (PR), Brasil.

\section{Rute Grossi Milani}

Doutora em Saúde Mental pela Universidade de São Paulo (USP); Docente Titular do Programa de Pós-graduação em Promoção da Saúde e Tecnologias Limpas Centro Universitário de Maringá (UNICESUMAR); Pesquisadora no Instituto Cesumar de Ciência, Tecnologia e Inovação (ICETI), Maringá (PR), Brasil.

E-mail: rute.milani@unicesumar.edu.br
RESUMO: O estudo buscou caracterizar o comportamento de uso das tecnologias digitais por jovens universitários e tecer apontamentos sobre as possíveis inferências em sua saúde. Participaram 361 jovens, com idade entre 17 e 24 anos, matriculados em uma instituição de ensino no norte do Paraná. Aplicou-se um questionário contendo questões sobre: idade do primeiro acesso, frequência e finalidade do uso. A análise estatística, por meio de regressão logística, mostrou que as tecnologias de informática são usadas para fins de trabalho ou jogo, nos períodos vespertino e noturno, por um intervalo de até quatro horas diárias, enquanto as de telefonia são usadas mais de oito horas, em todos os períodos do dia, para fins de relacionamentos sociais. Tais resultados mostram que elas estão imersas no cotidiano dos jovens, especialmente na dimensão afetiva e social, porém o uso prolongado pode indicar alterações em hábitos cruciais à saúde integral do jovem, como o sono e atividades físicas. Conclui-se que as tecnologias são um importante meio de interação e aprendizado dos jovens, o que deve ser explorado pelas políticas públicas para assegurar o cuidado ao desenvolvimento sadio da juventude.

PALAVRAS-CHAVE: Adulto jovem; Promoção da saúde; Tecnologia da informação.

\section{THE USE OF DIGITAL TECHNOLOGIES IN AN INSTITUTE OF HIGHER EDUCATION: NOTES FOR YOUNG PEOPLE'S HEALTH}

\begin{abstract}
The use of Digital Technologies by undergraduates is characterized and notes are elaborated on possible repercussions on their health. Three hundred and sixty-one young people, aged between 17 and 24 years, enrolled in an institute of higher education in the northern region of the state of Paraná, participated. A questionnaire on age at first access, frequency and aim was applied. Statistical analysis showed through logistic regression that information technologies are used for work or leisure, during the evening and night, for a period of four hours, whereas telephony is used for more than 8 hours at all times of the day for social relationship. Results show that technology lies within the day-to-day living of young people, especially within the affective and social life. However, their long use may indicate crucial habits, such as sleep and physical activities, harming young people's health. Technologies are important for interaction and learning and should be employed by public policies to ensure healthy development.
\end{abstract}

KEY WORDS: Young adult; Health promotion; Information Technology. 


\section{INTRODUÇão}

Os jovens estão sempre em busca de novidades e da aquisição de novos conhecimentos; querem conquistar o seu espaço e buscam serem aceitos por determinados grupos na sociedade; nasceram e cresceram em meio à revolução das tecnologias digitais e se adaptaram a essa realidade de forma tão natural, que os levou a serem considerados como nativos digitais.

A juventude é em geral caracterizada como um momento de introdução à vida adulta, fase na qual a maturidade psicológica vai depender da descoberta da própria identidade, da independência dos pais, do desenvolvimento de um sistema de valores e de relacionamentos (PAPALIA; FELDMAN, 2013). As transformações físicas e psicológicas vão conduzir o jovem a construir uma identidade e a implementar a socialização, assim, eles buscam a autonomia, o autocontrole e a responsabilidade.

A sociedade contemporânea segue o mesmo ritmo de desenvolvimento; com o surgimento das tecnologias observou-se que os comportamentos agora giram em torno da inovação, do aprendizado, da mudança e da competitividade. Yzer e Southwell (2008), destacam que novos estudos são necessários considerando a natureza multidisciplinar da relação entre as tecnologias e o homem. Para os autores, as tecnologias se configuram como um campo de vastas e rápidas mudanças, levando o usuário e as tecnologias a conviverem em um ambiente de constante transição. Assim, os jovens se comunicam nas redes por meio da tecnologia digital e expõe sentimentos, pensamentos e comportamentos influenciados pela mobilidade e conectividade (WEBER; SANTOS; CRUZ, 2014).

Moraes et al. (2015), afirmam que o uso das tecnologias digitais pode alterar alguns comportamentos dos jovens, bem como transformar o jeito deles pensarem, sentirem e agirem. A tecnologia melhora as habilidades de conhecimento, compreensão e muitas vezes é utilizada como artefato do aprender devido a gama de potencialidades de uso que ela permite, entretanto as experiências virtuais proporcionadas pelas tecnologias podem prejudicar as relações sociais pois, conforme vão crescendo e amadurecendo, criam novos laços de amizade cada vez mais em contexto virtual, fora do espaço de convivência e, como resultado, os laços com pessoas próximas podem tornar-se fragilizados, acarretando uma diminuição na comunicação (SILVA ROSADO; TOMÉ, 2015). Contudo, é possível identificar em alguns jovens um perfil mais desinibido, com liberdade de expressão, já que percebem as tecnologias digitais como uma forma de ampliar sua rede social $\mathrm{e}$ estarem em contato com diferentes pessoas em qualquer parte do mundo (NAVARRO; YUBERO, 2012). Elas também mudaram algumas noções básicas, tais como as de tempo e espaço; relações de consumo e a distinção entre o público e o privado; no entanto, possibilitaram a criação de realidades virtuais que podem levar o ser humano a experiências online muito parecidas com experiências reais (SOUZA, 2014).

Dentre as tecnologias digitais, consideram-se as tecnologias de uso pessoal, tais como, o computador de mesa e o portátil, e o aparelho celular. Essas tecnologias são reconhecidas como extremamente necessárias à pessoa na contemporaneidade, como forma de comunicação e de instrumentalização do indivíduo em relação ao meio em que está inserido (PALHARES, 2001). Entretanto, salienta-se que o uso das tecnologias digitais possui um impacto sobre a saúde do jovem e sua maneira de interagir e agir com o ambiente, podendo ocorrer transformações sociais, além do risco de dependência de jogos eletrônicos, isolamento diante da sociedade e o distanciamento do real (LEMOS; SANTANA, 2011; FRAGA; SILVA, 2010; MARÇAL; MELLO; SOUZA CORRÊA, 2012). Além de aspectos sociais, características físicas podem estar associadas, como o sedentarismo, o distúrbio do sono, ansiedade, depressão, cansaço, irritabilidade, perda da memória e tontura (ENES; SLATER, 2010; MESQUITA; REIMÃO, 2007; MESQUITA; REIMÃO, 2010; SILVA et al., 2015).

Considerando que essas tecnologias se incorporam a cada dia com mais intensidade no cotidiano das pessoas, é de conhecimento de todos que o seu uso traz transformações, mudanças comuns e até mesmo radicais na construção da subjetividade; desse modo, surge então a necessidade de conhecer esses fenômenos relacionais entre o sujeito e a tecnologia (NICOLACI-DACOSTA, 2002). Pois, essa nova relação tem transformado 
os tipos de interações possíveis, surgindo assim uma nova forma de cultura, manifestada no meio virtual por meio de palavras escritas na rede interligando diferentes pessoas (FRAGA; SILVA, 2010).

Neste estudo busca-se caracterizar o comportamento de uso das tecnologias digitais por jovens universitários, a finalidade e a frequência de uso no cotidiano, e discutir seu impacto na saúde.

\section{MÉTODO}

A pesquisa é caracterizada por ser um estudo exploratório e transversal, segundo Gil (2008), essa metodologia é caracterizada pela delimitação de um objeto novo a ser investigado e, por meio de informações iniciais sobre a temática, incentivando novas pesquisas e intervenções.

Participaram da pesquisa 361 jovens de ambos os sexos, dos quais 206 do sexo feminino e 155 do sexo masculino, com idades entre 17 e 24 anos, todos estudantes do $1^{\circ}$ ano do ensino superior e matriculados em uma instituição de ensino privado no interior do Paraná; o critério de inclusão dos participantes foi estabelecido com base na idade, representando o período de transição para a fase adulta; todos assinaram o termo de consentimento livre e esclarecido, aos menores de 18 anos solicitou-se aos pais ou responsáveis, previamente, a assinatura do termo. O tempo médio de coleta de dados foi de aproximadamente 20 minutos, realizada no início ou final das aulas. A pesquisa ocorreu de acordo com a apreciação e aprovação da pesquisa junto ao Comitê de Ética do Centro Universitário de Maringá, sob o parecer $\mathrm{n}^{\mathrm{o}} 379.115$.

A coleta de dados aconteceu em sala de aula a partir da aplicação de um questionário, elaborado pelos autores, baseado no questionário de Hábitos de Lazer, sub item adolescência (VERMELHO, 2003), com o objetivo de identificar as atitudes dos jovens em relação à tecnologia digital. $\mathrm{O}$ instrumento foi dividido em duas etapas uma que se referia ao comportamento de descarte das tecnologias, composto por dez questões, e outra sobre o comportamento de uso, composto por 10 questões, foi dividido em três blocos, o primeiro a respeito da identificação do sujeito; o segundo sobre o perfil de acesso às tecnologias digitais, englobando a idade do primeiro acesso e a frequência do uso, tanto por períodos do dia como horas de uso; e o terceiro referente à identificação quanto à finalidade do uso.

Antes de proceder a descrição da análise de dados empregada, algumas considerações são necessárias, visto que a delimitação do público alvo voltado somente aos jovens gerou questionamentos quanto ao uso das tecnologias nas demais fases do desenvolvimento humano, tais como infância, vida adulta e terceira idade. Outra limitação do estudo é relativa ao questionário, o qual, em algumas questões, não permitiu avaliar valores, resultando em mais de uma resposta por questão, classificadas em mais de uma categoria, portanto, recomenda-se o uso de escalas e entrevistas em profundidade para exploração das demais variáveis relacionadas ao uso das tecnologias.

Nas questões foram consideradas as tecnologias do tipo computador, notebook e aparelhos celulares. Foram atribuídos às respostas dadas pelos participantes valores iguais a " 1 " para uma resposta assinalada e "0" (zero) caso contrário. A respeito da finalidade do uso, os participantes poderiam responder a mais de uma alternativa por questão, o que pode implicar em um percentual superior a $100 \%$ nos resultados. Além disso, adotou-se a categorização "1" e "0" para representar jovens do sexo "feminino" e "masculino", respectivamente.

Para a análise de dados, inicialmente foi feita uma análise exploratória, pois, considerando que o questionário buscava levantar informações sobre o uso das tecnologias, foi preciso averiguar se os dados obtidos eram significantes para afirmar a maneira pela qual se dá o uso das tecnologias; em seguida, ajustaram-se oito modelos de regressão logística (Equação 1) para o uso de tecnologias de informática e telefonia, considerando cada um dos períodos em análise, descritos a seguir: matutino, vespertino, noturno e madrugada.

$$
\log \operatorname{it}(\mathrm{p})=\ln \left(\frac{\mathrm{p}}{1-\mathrm{p}}\right)=\beta_{0}+\sum_{\mathrm{i}=1}^{\mathrm{k}} \beta_{\mathrm{i}} \mathrm{X}_{\mathrm{i}}
$$

Para melhor compreensão da análise, segue um exemplo referente ao período matutino, em que a 
frequência de uso da tecnologia foi considerada como variável resposta $(Y)$ e dois modelos foram ajustados: um em que $P=P(Y=1)$ representa a probabilidade de que a maior frequência de uso da tecnologia de informática ocorreu no período matutino e $1-\mathrm{p}=\mathrm{P}(\mathrm{Y}=0)$ a probabilidade de que a maior frequência de uso da tecnologia não ocorreu no período matutino; e o outro similar, mas para o uso de tecnologias de telefonia.

As variáveis explicativas do modelo representadas por $\mathrm{X}$ relacionaram-se à finalidade de uso das tecnologias. As análises foram feitas utilizando-se o ambiente estatístico R e o nível de significância ( $\alpha$ ) adotado foi igual a $5 \%$.

\section{RESULTADOS}

A tabela 1 apresenta o percentual de respostas dos jovens segundo o tipo de tecnologia digital e a idade do primeiro acesso.

Tabela 1. Tipo de tecnologia e a idade do primeiro acesso

\begin{tabular}{ccccc}
\hline $\begin{array}{c}\text { Idade } \\
(\text { anos })\end{array}$ & $\begin{array}{c}\text { Computador } \\
(\%)\end{array}$ & $\begin{array}{c}\text { Notebook } \\
(\%)\end{array}$ & $\begin{array}{c}\text { Smartphone } \\
(\%)\end{array}$ & $\begin{array}{c}\text { Celular } \\
(\%)\end{array}$ \\
\hline $5-9$ & 22 & 3 & 0 & 7 \\
$9-14$ & 57 & 14 & 2 & 64 \\
$14-19$ & 17 & 63 & 51 & 26 \\
$19-25$ & 0 & 12 & 16 & 0 \\
\hline
\end{tabular}

Fonte: Dados da pesquisa

É possível identificar que mais da metade dos jovens manifestaram que o primeiro acesso à tecnologia do tipo computador (57\%) e celular (64\%) deu-se entre os 9 e 14 anos, e entre os 14 e 19 para o notebook (63\%) e smartphone (51\%). Ressalta-se que $22 \%$ dos jovens alegaram o primeiro acesso ao computador com idades entre 5 e 9 anos. Entretanto, $4 \%$ das pessoas pesquisadas não informaram a idade de primeiro acesso ao computador, $8 \%$ não informaram para notebook, $31 \%$ para smartphone e 3\% para o celular.

$\mathrm{Na}$ tabela 2 está disposto o percentual de respostas assinaladas pelos jovens de acordo com o período do dia em que mais utilizam suas tecnologias.
Tabela 2. Períodos de uso das tecnologias digitais

\begin{tabular}{lccc}
\hline Tecnologia & Período & $\begin{array}{c}\text { Masculino } \\
\text { \% }\end{array}$ & $\begin{array}{c}\text { Feminino } \\
\%\end{array}$ \\
\hline \multirow{3}{*}{ Informática } & Manhã & 16 & 22 \\
& Tarde & 35 & 45 \\
& Noite & 32 & 46 \\
& Madrugada & 21 & 24 \\
& & & \\
Telefonia & Manhã & 31 & 43 \\
& Tarde & 38 & 51 \\
& Noite & 35 & 46 \\
\hline
\end{tabular}

Fonte: Dados da pesquisa

É fato que os jovens permanecem boa parte dos seus dias conectados a alguma de suas tecnologias digitais, porém, o período do dia que eles mais utilizam suas tecnologias de informática seria durante a tarde $\mathrm{e}$ a noite tanto para o sexo masculino como para o sexo feminino. Tais afirmações podem ser confirmadas observando a Tabela 3 a qual irá apresentar o percentual de jovens segundo o tempo médio de uso das tecnologias digitais no período de um dia.

Tabela 3. Tempo médio de uso dos equipamentos

\begin{tabular}{lcccc}
\hline $\begin{array}{l}\text { Tempo } \\
\text { Médio }\end{array}$ & $\begin{array}{c}\text { Computador } \\
\%\end{array}$ & $\begin{array}{c}\text { Notebook } \\
\%\end{array}$ & $\begin{array}{c}\text { Smartphone } \\
\%\end{array}$ & $\begin{array}{c}\text { Celular } \\
\%\end{array}$ \\
\hline $\begin{array}{l}\text { Não Utilizam } \\
\text { Até } 1 \text { hora }\end{array}$ & 31 & 15 & 36 & 27 \\
$\begin{array}{l}1 \text { hora a } 4 \\
\text { horas }\end{array}$ & 24 & 41 & 11 & 12 \\
$\begin{array}{l}4 \text { horas a } 8 \\
\text { horas }\end{array}$ & 21 & 27 & 17 & 16 \\
$\begin{array}{l}\text { Mais de } 8 \\
\text { horas }\end{array}$ & 13 & 11 & 32 & 37 \\
\hline
\end{tabular}

Fonte: Dados da pesquisa

Observa-se que $24 \%$ dos jovens declararam fazer uso do computador em um período de 1 a 4 horas, assim como 41\% para o notebook. É possível observar também que $32 \%$ dos jovens utilizam em média seu smartphone por mais de 8 horas e $37 \%$ o aparelho celular também por mais de 8 horas. A tabela 4 apresenta as estimativas dos parâmetros dos modelos de regressão logística, 
significativas a 5\%, para cada período de utilização (manhã, tarde, noite e madrugada) com os respectivos valores p, estimativas de odds ratio e limites de $95 \%$ de confiança, tanto para as tecnologias de informática como para as tecnologias de telefonia.

Tabela 4. Modelo de regressão logística

\begin{tabular}{|c|c|c|c|c|c|c|}
\hline Tecnologia & Período/Finalidade & $\hat{\beta}$ & Valor $\mathrm{p}$ & $O R$ & $\mathrm{LI}$ & LS \\
\hline \multicolumn{7}{|c|}{ Manhã } \\
\hline & Atividades/trabalho & 0,70 & 0,011 & 2,004 & 1,170 & 3,432 \\
\hline & Jogos Variados & 0,50 & 0,030 & 1,649 & 1,048 & 2,596 \\
\hline & Redes Sociais & 0,52 & 0,028 & 1,691 & 1,056 & 2,708 \\
\hline \multicolumn{7}{|c|}{ Tarde } \\
\hline Informática & Pesquisas Escolar & 0,98 & 0,009 & 2,667 & 1,270 & 5,601 \\
\hline & Escutar Música & 0,73 & 0,009 & 2,081 & 1,197 & 2,081 \\
\hline \multicolumn{7}{|c|}{ Noite } \\
\hline & Relações Afetivas & 0,64 & 0,016 & 1,898 & 1,127 & 3,196 \\
\hline \multicolumn{7}{|c|}{ Madrugada } \\
\hline & Jogos Variados & 0,71 & 0,008 & 2,052 & 1,347 & 3,127 \\
\hline \multicolumn{7}{|c|}{ Manhã } \\
\hline & Pesquisa Escolar & 0,84 & 0,029 & 2,322 & 1,088 & 4,951 \\
\hline & Redes Sociais & 1,57 & $<, 0001$ & 4,788 & 2,881 & 7,955 \\
\hline \multicolumn{7}{|c|}{ Tarde } \\
\hline & Pesquisas Diversas & 1,00 & 0,070 & 2,721 & 1,169 & 6,336 \\
\hline & Redes Sociais & 0,89 & 0,009 & 2,441 & 1,245 & 4,784 \\
\hline \multirow[t]{7}{*}{ Telefonia } & \multicolumn{6}{|l|}{ Noite } \\
\hline & Relações Afetivas & 0,76 & 0,007 & 2,142 & 1,228 & 3,737 \\
\hline & Redes Sociais & 1,32 & $<, 0001$ & 3,775 & 2,149 & 6,630 \\
\hline & \multicolumn{6}{|l|}{ Madrugada } \\
\hline & Relações Afetivas & 0,62 & 0,013 & 1,862 & 1,140 & 3,041 \\
\hline & Escutar Música & 0,53 & 0,037 & 1,698 & 1,032 & 2,793 \\
\hline & Redes Sociais & 0,60 & 0,018 & 1,824 & 1,106 & 3,008 \\
\hline
\end{tabular}

Fonte: Dados da pesquisa

As variáveis significativas no modelo diferiram de período para período. Por exemplo, o modelo ajustado para o período da madrugada foi $\log \left(\frac{\mathrm{p}}{1-\mathrm{p}}\right)=0,71 \mathrm{X}_{1}$ em que, $X_{1}$ representa a utilização da tecnologia de informática para jogos no período da madrugada. Por outro lado, para todos os períodos, os intervalos de confiança não contêm o valor 1 , isto significa que as variáveis de uso no período da manhã, da tarde, da noite e da madrugada são consideradas fatores que apresentam maior chance de ocorrência para os respectivos modelos. Ressalta-se que os interceptos $\left(\hat{\beta}_{0}\right)$ não foram significativos a $5 \%$, por isso, a não inclusão do termo em todos os modelos. 
As estimativas das probabilidades que cada jovem tem de fazer uso das tecnologias em função das variáveis do modelo fornecem informações sobre os jovens isoladamente. Em relação aos equipamentos de informática, verifica-se que a chance predita do período matutino ser o horário de maior utilização para os jovens que utilizam com a finalidade de atividades do trabalho ou faculdade é duas vezes maior em comparação aos que não utilizam para essa finalidade; para os que utilizam para jogar é 64\% maior no período matutino; e para os que utilizam para redes sociais é $69 \%$ maior nesse período do dia.

Com relação ao período vespertino, a chance de o jovem utilizar sua tecnologia de informática para realizar pesquisas escolares é duas vezes maior, assim como para escutar música. Por outro lado, durante a madrugada, há duas vezes mais chance do jovem utilizála para jogar. Diante disso, é possível identificar que os jovens fazem uso dos aparelhos de informática mais para atividades de trabalho ou faculdade, realizando pesquisas diversas e, para diversão, por meio de jogos variados ou, até mesmo, pra ouvir músicas e navegar nas redes sociais.

Referente à tecnologia de telefonia, o modelo de regressão logística indica que a chance predita para os jovens usarem suas tecnologias de telefonia em redes sociais no período matutino é 4 vezes maior, no período vespertino é 2 vezes, e no noturno é 3 vezes maior. Pode-se afirmar que as tecnologias de telefonia são mais utilizadas pelos jovens para suas relações afetivas, para estarem em suas redes sociais e realizar pesquisas diversas, ou seja, configurando um uso mais voltado para as relações sociais.

\section{DISCUSSÃO}

Ao analisar a idade de primeiro acesso das tecnologias pelos jovens foi possível identificar que o uso se deu ainda na infância, período no qual a criança está em processo de desenvolvimento cognitivo e da personalidade. $\mathrm{O}$ uso das tecnologias digitais nessa fase está muitas vezes associado ao modismo, à busca por novidade e por ser uma ferramenta de socialização; ou ainda pela confiança encontrada por eles na internet para se expressarem com qualidades e defeitos, o que facilita o processo de aceitação de si (ROMÃO-DIAS; NICOLACI-DA-COSTA, 2012). O ambiente virtual é atrativo e permite novas tendências de organizações sociais, tal realidade deve ser enfatizada, no sentido de suportar as virtualidades como vivências, uma vez que nas redes existem a colaboração, o compartilhamento e a comunicação como possibilidades humanas, mediadas por um objeto (BAUMAN, 2008). Desse modo, estar conectados a maior parte do tempo é percebido por eles como algo natural, pois estão se desenvolvendo e se transformando concomitantemente às tecnologias em busca de autonomia, autoestima e identidade (GROSSI et al., 2014).

Os dados que se referiram ao período do dia que mais utilizam as tecnologias comprovam que os jovens permanecem conectados boa parte do tempo a mais de uma tecnologia. Com o surgimento das tecnologias digitais, os meios de comunicação se tornaram interativos, houve uma quase extinção das limitações de tempo e espaço, de modo que a comunicação passou a ser mais flexível, instantânea e interativa, com um clique pode se acessar a qualquer informação e ainda manter contato com uma pessoa distante fisicamente (VERMELHO et al., 2014).

Assim, é possível compreender quais são os motivos que levam os jovens a passarem boa parte do dia ligados a uma de suas tecnologias e que vem ao encontro do apresentado por Souza (2014) que relata ser comum ocorrer o uso simultâneo dos recursos tecnológicos; pode-se jogar com amigos no computador e falar com outros no celular, entretanto deve-se ter cautela pois, pode resultar em uma redução do autocontrole, risco de dependência da tecnologia e diminuição do desempenho. Ressalta-se, conforme Valente (2014), como as tecnologias concentram em um único aparelho a possibilidade de uso de várias funcionalidades, elas estão sempre às mãos dos jovens e estes não precisam ser especialistas para utilizá-las, permitindo que eles sejam autores, produtores e disseminadores do conhecimento. As vivências entre as diferentes funcionalidades e a adaptação frente às tecnologias digitais, culminaram na terminologia "Imigrante digital" expressa por Prensky (2001) para diferenciar o nativo digital, daqueles 
que necessitam se adequar e aprender a utilizar tais ferramentas, estes processos resultam na diferença marcante entre gerações, mesmo em um curto espaço de tempo (SOUZA; PAULA CORREIA; SOUZA, 2015).

A presente pesquisa revelou que mais de $30 \%$ dos jovens pesquisados fazem uso das tecnologias de telefonia por mais de 8 horas diárias, em praticamente todos os períodos do dia, isso levanta preocupações sobre os possíveis efeitos à saúde decorrentes do uso intensivo. Silva et al. (2015), em pesquisa com pessoas residentes próximo à estação radiobase de telefonia celular, estudaram a associação entre exposição a radiação eletromagnética não ionizante, encontrada em aparelhos celulares, à saúde mental. Os autores relataram que a absorção da irradiação mostrou-se associada a efeitos diversos, tais como: sintomas depressivos; sintomas de ansiedade; palpitações por ter o telefone perto do corpo na maior parte do tempo e sintomas de irritabilidade. Portanto, o uso das tecnologias digitais por longos períodos, conforme observado neste estudo, deve ser analisado com cautela.

O uso noturno das tecnologias digitais, também pode representar impactos na saúde humana, principalmente referente à qualidade do sono, visto que o sono supre necessidades fisiológicas e psicológicas, na adolescência sua regulação é crucial, dada a conexão entre sono, memória e aprendizagem. No período noturno, as funções multitarefas, atrelada às condições de privacidade, fornecida pelo ambiente do quarto, por exemplo, propicia o jovem a permanência na tecnologia por horas, adentrando a madrugada e "esquecendo-se" de dormir. Em casos mais graves, podem ainda apresentar problemas crônicos, como a sonolência diurna excessiva, além de desdobramentos em suas interações sociais (AZEVEDO, 2016).

Nesta investigação os jovens pesquisados apresentaram comportamento diferenciado quanto ao tipo de tecnologia, sendo as tecnologias de informática, computadores e notebooks utilizados para fins de trabalho ou jogos, geralmente em um período de até 4 horas diárias; enquanto as tecnologias de telefonia são usadas para fins de relacionamentos interpessoais, como troca de mensagens instantâneas e uso de redes sociais por mais de oito horas por dia. Tais mudanças possibilitam que as diferenças entre os jovens possam ser percebidas, valorizadas e aceitas, facilitando a auto expressão (MAYORGA, 2006). Os recursos interativos disponibilizados pelas tecnologias digitais permitem que todos os usuários sejam ativos na produção coletiva de conhecimentos e compartilhamentos, de modo que qualquer um pode ser difusor de conhecimento, informação e saber (GROSSI et al., 2014). As pessoas possuem agora, por meio das redes, mais liberdade de expressão, participam ativamente na troca de informações e mobilizações, porém, consequentemente, surge um desafio para os profissionais da comunicação que devem apresentar constantemente experiências empolgantes para o público (VERMELHO et al., 2014).

\section{CONCLUSÃO}

As tecnologias digitais de informática e de telefonia mostraram-se um importante meio para as trocas nas relações interpessoais e vivências entre os jovens pesquisados, entretanto, o uso exagerado representa possíveis impactos na saúde dos jovens, como na qualidade do sono. Sugerimos que pesquisas futuras se atenham às implicações que o uso das tecnologias digitais tem trazido para a saúde, ao comportamento e às relações sociais, enfim, às novas configurações subjetivas que permeiam a relação do jovem com o mundo virtual, pois as tecnologias são um importante meio de intermediação com as políticas públicas para assegurar o cuidado ao desenvolvimento sadio da juventude e devemos aproveitar desse meio para explorar potencialidades e habilidades que podem ser desenvolvidas com seu uso.

\section{AGRADECIMENTOS}

Ao Programa de Bolsas de Iniciação Científica do Cesumar (PROBIC), Centro Universitário de Maringá (UNICESUMAR), pelo apoio financeiro. 


\section{REFERÊNCIAS}

AZEVEDO, D.P.G.D.; AZEVEDO, N.G. A relação do sonoaprendizagem e as novas tecnologias de informação e comunicação: um desafio na educação dos adolescentes. In: ENCONTRO VIRTUAL DE DOCUMENTAÇÃO EM SOFTWARE LIVRE E CONGRESSO INTERNACIONAL DE LINGUAGEM E TECNOLOGIA ONLINE, 2016. Anais... Belo Horizonte: UFMG, 2016.

BAUMAN, Z. Vida para consumo: a transformação de pessoas em mercadoria. Rio de Janeiro, Zahar, 2008.

ENES, C. C.; SLATER, B. Obesidade na adolescência e seus principais fatores determinantes. Rev. bras. epidemiol., São Paulo, v. 13, n. 1, p. 163-171, mar. 2010.

FRAGA, E.A.M.; DA SILVA, C. L.. Comunidades virtuais de internet: Atualização do debate sobre lazer. LICERERevistadoProgramadePós-graduaçãoInterdisciplinar em Estudos do Lazer, v. 13, n. 4, 2010.

GIL, A. C. Métodos e técnicas de pesquisa social. 6. ed. São Paulo: Atlas, 2008.

GROSSI, M. G. R. et al. A utilização das tecnologias digitais de informação e comunicação nas redes sociais pelos universitários brasileiros. Texto Digital, v. 10, n. 1, p. $4-23,2014$.

LEMOS, I. L.; SANTANA, S. M. Dependência de jogos eletrônicos: a possibilidade de um novo diagnóstico psiquiátrico. Rev Psiq Clín, v. 39, n. 1, p. 28-33, 2011.

MARÇAL, M.C.C.; DE MELLO, S.C.B.; DE SOUZA CORRÊA, M. I.. As crises silenciadas pela modernidade e pelas tecnologias da cultura da virtualidade real1/The silenced crisis of modernity and culture technologies of real virtuality. Rev FAmecos, v. 19, n. 1, p. 249, 2012.

MAYORGA, C. Identidades e adolescências: uma desconstrução. Pesquisas e Práticas Psicossociais, v. 1, n. 1, p. 1-20, 2006.

MESQUITA, G.; REIMÃO, R. Nightly use of computer by adolescents: its effect on quality of sleep. Arq Neuropsiquiatr, v. 65, n. 2B, p. 428-432, 2007.

MESQUITA, G.; REIMÃO, R.. Quality of sleep among university students: effects of nighttime computer and television use. Arq Neuropsiquiatr, v. 68, n. 5, p. 720$725,2010$.
MORAES, M. C. et al. As tecnologias digitais na geometria do cotidiano: o (re) pensar da prática docente. Revista PRISMA.COM, n. 28, 2015.

NAVARRO, R.; YUBERO, S. Impacto de la ansiedad social, las habilidades sociales y la cibervictimización en la comunicación online. Escr Psicol (Internet), v. 5, n. 3, p. 4-15, 2012.

NICOLACI-DA-COSTA, A.M. Revoluções tecnológicas e transformações subjetivas. Psicologia: teoria e pesquisa, v. 18, n. 2, p. 193-202, 2002.

PALHARES, I. O consumo de tecnologia no rastro da aceleração da obsolescência. ComCiência, n. 131, p. 0-0, 2011.

PAPALIA, D.E.; FELDMAN, R.D. Desenvolvimento humano. Porto Alegre, RS, Artmed, 2013.

PRENSKY, M. Digital natives, digital immigrants part 1. On the horizon, v. 9, n. 5, p. 1-6, 2001.

ROMÃO-DIAS, D.; NICOLACI-DA-COSTA, A. M. O brincar e a realidade virtual. Cadernos de psicanálise (Rio de Janeiro), v. 34, n. 26, p. 85-101, 2012.

SILVA, D. F. et al. Exposure to non-ionizing electromagnetic radiation from mobile telephony and the association with psychiatric symptoms. Cad. Saúde Pública, Rio de Janeiro, v. 31, n. 10, p. 2110-2126, out. 2015.

SILVA ROSADO, L. A.; TOMÉ, V. M. N. As redes sociais na internet e suas apropriações por jovens brasileiros e portugueses em idade escolar. Rev Bras Estudos Pedagógicos, v. 96, n. 242, 2015.

SOUZA, J.A.S. A nova era digital e as performances do corpo. ARTEFACTUM-Revista de estudos em Linguagens e Tecnologia, v.1, n.1, 2014.

SOUZA, M.; DE PAUlA CORREIA, V. G.; DE SOUZA, C. H. M.. O real nativo e imigrante digital nas redes sociais digitais. Inter Science Place, v. 1, n. 24, 2015.

VALENTE, J.A.A comunicação e a educação baseada no uso das tecnologias digitais de informação e comunicação. UNIFESO-Humanas e Sociais, v. 1, n. 1, p. 141-166, 2014.

VERMELHO, S. C. et al. Refletindo sobre as redes sociais digitais. Educação \& Sociedade, v. 35, n. 126, 2014. 
VERMELHO, S. C. Educação e virtualização: as mídias e a formação do individuo. São Paulo: PUCSP, Programa de Pós Graduação em Educação, 2003.

WEBER, A.; SANTOS, E.; DA CRUZ, M.M. Letramentos e alfabetizações na cibercultura: crianças e jovens em rede, desafios para educação. Leitura: Teoria \& Prática, v. 32, n. 62 , p. $59-73,2014$

YZER, M.C.; SOUTHWELL, B.G. New communication technologies, old questions. Am Behav Sci., v. 52, n. 1, p. 8-20, 2008.

Recebido em: 26 de setembro de 2016 Acesso em: 21 de fevereiro de 2017 